\title{
MAGNETO HYDRODYNAMIC FLOW WITH VISCOUS DISSIPATION EFFECTS IN THE PRESENCE OF SUCTION AND INJECTION
}

\author{
M. Chandrasekar, M.S. Kasiviswanathan \\ Department of Mathematics, Anna University, Chennai, India \\ e-mail:mchandru@annauniv.edu; mskasi@gmail.com
}

\begin{abstract}
Gyarmati's variational principle developed on the thermodynamic theory of irreversible processes is employed to study the viscous dissipation effects with uniform suction and injection on the infinite flat plate. The velocity and temperature fields inside the boundary layer are approximated as simple polynomial functions, and the functional of the variational principle is constructed. The Euler Lagrange equations are reduced to simple polynomial equations in terms of velocity and thermal boundary layer thicknesses. The velocity, temperature profiles, skin friction and heat transfer with the viscous dissipation effects are analyzed and are compared with known numerical solutions. The comparison of the present solution with the existing solutions establishes the fact that the accuracy is remarkable.
\end{abstract}

Keywords: irreversible processes, velocity and temperature functions, viscous dissipation, suction and injection

\section{Introduction}

The prime objective of this investigation is to study the effects of viscous dissipation on the magneto hydrodynamic flow over a semi infinite flat plate with uniform suction and injection by using some recent developments in the field of thermodynamics of irreversible processes and to obtain numerical solution to the boundary layer flow and heat transfer with the help of a variational technique based on the governing principle of dissipative processes. According to the boundary layer theory, the irreversible processes of momentum and heat transfer in flows around bodies occur mainly inside a very thin layer adjacent to the surface of the body. Hence, it is quite appropriate to study these non equilibrium processes by a variational technique developed in the field of irreversible thermodynamics.

The boundary layer flow of an incompressible electrically conducting fluid past a semi infinite flat plate in the presence of a transverse magnetic field has been studied recently by many researchers. The boundary layer solution for the magneto hydrodynamic flow over a semi infinite flat plate in the presence of transverse magnetic field was studied by Watanabe $(1978,1986)$ and Watanabe and Pop (1994) by means of a difference-differential method. The analysis on stagnation point flow and asymmetric flow was investigated by Sparrow et al. (1963), Ariel (1994), Raptis (1991) and Chamkha (1998). Watanabe (1986) analyzed the magneto hydrodynamic boundary layer flow over a wedge and did not considered the energy equation. Hossain (1992) treated the viscous and Joule heating effects on magneto hydrodynamic free convection boundary layer flow with variable temperature on the plate. Watanabe and Pop (1993) solved the problem of magneto hydrodynamic free convection flow over a wedge in the presence of a transverse magnetic field. Soundalgekar and Takhar (1977) considered the boundary layer equations for the aligned flow and temperature of an electrically conducting fluid past a semi infinite heated flat plate in the presence of a transverse magnetic field. 
Yih (1998) presented an analysis for forced convection boundary layer flow over a wedge with uniform suction/blowing whereas Watanabe (1990) investigated the behavior of the boundary layer over a wedge with suction/injection in forced flow. Also Yih (1999) extended the work of Watanabe and Pop (1994) to investigate the heat transfer characteristics in magneto hydrodynamic forced convection flow adjacent to a non isothermal wedge in the presence of a transverse magnetic field. An approximate numerical solution for thermal stratification on magneto hydrodynamic steady laminar boundary layer flow over a wedge with suction/injection was investigated by Anjali Devi and Kandasamy (2003). Lin and Lin (1987) proposed a similarity solution method that provides accurate solutions for laminar forced convection heat transfer for either an isothermal surface or an uniform flux boundary to fluid of any Prandtl number. Ingham (1979) proved that the terms representing viscous dissipative heat and stress work are of equal importance in the case air and hence they should be both considered or neglected in the energy equation.

By considering all the above facts, in this study the non similar boundary layer flow with uniform suction and injection over a semi infinite flat plate in the presence of constant magnetic flux density is analyzed. Gyarmati's variational technique has been employed to solve the non similar boundary layer equation. The computational results are given for the velocity profile, temperature profile, the coefficient of skin friction (shear stress) and the Nusselt number (heat transfer) for various values of the suction and injection parameter $H$ and the magnetic parameter $\xi$. The present results are compared with known results and are found to be excellent. The intention of this investigation is to establish that Gyarmati's variational technique is one of the most general and exact variational techniques in solving heat transfer problems. Chandrasekar $(1998,2003)$, Chandrasekar and Baskaran $(2006,2007)$ has already applied Gyarmati's variational technique for steady and unsteady heat transfer and boundary layer flow problems.

\section{The governing equations and boundary conditions}

The system of steady, two dimensional, incompressible and laminar boundary layer flow over a semi infinite flat plate with suction and injection is considered. The leading edge of the plate is at $x=0$, the plate is parallel to the $x$-axis and infinitely long downstream. It is assumed that the flow is with a free stream velocity $U_{\infty}$ and the ambient temperature $T_{\infty}$ which are parallel to the $x$-axis. The boundary layer equations for the present system are considered with the usual boundary layer approximation with the assumption that all fluid properties are constants and are as follows

$$
\begin{aligned}
& u_{x}+v_{y}=0 \quad \text { (mass) } \\
& u u_{x}+v u_{y}=\nu u_{y y}+\frac{\kappa B_{0}^{2}}{\rho}\left(U_{\infty}-u\right) \quad(\text { momentum }) \\
& u T_{x}+v T_{y}=\alpha T_{y y}+\frac{\nu}{C_{p}}\left(u_{y}\right)^{2}-\frac{\kappa B_{0}^{2}}{\rho C_{p}} u\left(U_{\infty}-u\right) \quad \text { (energy) }
\end{aligned}
$$

subject to the boundary conditions

$$
\begin{aligned}
& y \rightarrow 0 \quad \Rightarrow \quad u=0, \quad v=v_{0}, \quad T=T_{0} \\
& y \rightarrow \infty \quad \Rightarrow \quad u=U_{\infty}=\text { const }, \quad T=T_{\infty}
\end{aligned}
$$

Here $u, v, v_{0}, U_{\infty}, T, T_{0}$ and $T_{\infty}$ are the velocity of the fluid in the $x$-direction, $y$-direction, suction/injection velocity, free stream velocity, temperature of the fluid, temperature of the plate and ambient temperature respectively. The symbols $\nu, \kappa, \rho, B_{0}, \alpha, C_{p}$ are respectively the kinematic viscosity, electrical conductivity, density, externally imposed magnetic field in the 
$y$-direction, thermal diffusivity and specific heat at constant pressure of the fluid. It is assumed that the temperature of the plate $T_{0}$ is greater than the ambient temperature $T_{\infty}$.

\section{Gyarmati's variational principle}

Gyarmati $(1969,1970)$ introduced a genuine variational principle called the Governing Principle of Dissipative Processes (GPDP) which is given in its universal form

$$
\delta \int_{V}(\sigma-\psi-\Phi) d V=0
$$

Principle (3.1) is valid for linear, quasi-linear and certain types of non-linear transport processes at any instant of time under constraints that the balance equations

$$
\rho \dot{a}_{i}+\nabla \cdot \mathbf{J}_{i}=\sigma_{i} \quad(i=1,2,3, \ldots, f)
$$

are satisfied. In Eq. (3.1), $\delta$ is the variational symbol, $\sigma$ is the entropy production, $\psi$ and $\Phi$ are dissipation potentials and $V$ is the total volume of the thermodynamic system. In Eq. (3.2), $\rho$ is the mass density and $\dot{a}, \mathbf{J}_{i}, \sigma_{i}$ are respectively substantial variation, flux and source density of the $i$-th extensive transport quantity $a_{i}$. The entropy production $\sigma$ per unit volume and unit time can always be written in the bilinear form

$$
\sigma=\sum_{i=1}^{f} \mathbf{J}_{i} \cdot \mathbf{X}_{i} \geqslant 0
$$

where $\mathbf{J}_{i}$ and $\mathbf{X}_{i}$ are fluxes and forces respectively. According to Onsagar's linear theory (Onsagar, 1931), the fluxes are linear functions of forces, that is

$$
\mathbf{J}_{i}=\sum_{k=1}^{f} L_{i k} \mathbf{X}_{k} \quad(i=1,2,3, \ldots, f)
$$

or alternatively

$$
\mathbf{X}_{i}=\sum_{k=1}^{f} R_{i k} \mathbf{J}_{k} \quad(i=1,2,3, \ldots, f)
$$

The constants $L_{i k}$ and $R_{i k}$ are conductivities and resistances respectively satisfying the reciprocal relations (Onsagar, 1931)

$$
L_{i k}=L_{k i} \quad R_{i k}=R_{k i} \quad(i, k=1,2,3, \ldots, f)
$$

The matrices of $L_{i k}$ and $R_{i k}$ are mutually reciprocals and they are symmetric, that is

$$
\sum_{m=1}^{f} L_{i m} R_{m k}=\sum_{m=1}^{f} L_{m k} R_{i m}=\delta_{i k} \quad(i, k=1,2,3, \ldots, f)
$$

where $\delta_{i k}$ is the Kronecker delta. The local dissipation potentials $\psi$ and $\Phi$ are defined as (Onsagar, 1931)

$$
\psi(\mathbf{X}, \mathbf{X})=\frac{1}{2} \sum_{i, k=1}^{f} L_{i k} \mathbf{X}_{i} \cdot \mathbf{X}_{k} \geqslant 0 \quad \Phi(\mathbf{J}, \mathbf{J})=\frac{1}{2} \sum_{i, k=1}^{f} R_{i k} \mathbf{J}_{i} \cdot \mathbf{J}_{k} \geqslant 0
$$


In the case of transport processes, the forces $\mathbf{X}_{i}$ can be generated as gradients of certain $\Gamma$ variables and can be written as

$$
\mathbf{X}_{i}=\nabla \Gamma_{i}
$$

Principle (3.1) with the help of Eqs. (3.3), (3.8) and (3.9) takes the form

$$
\delta \int_{V}\left(\sum_{i=1}^{f} \mathbf{J}_{i} \cdot \nabla \Gamma_{i}-\frac{1}{2} \sum_{i, k=1}^{f} L_{i k} \nabla \Gamma_{i} \cdot \nabla \Gamma_{k}-\frac{1}{2} \sum_{i, k=1}^{f} R_{i k} \mathbf{J}_{i} \cdot \mathbf{J}_{k}\right) d V=0
$$

This variational principle has been already applied to various dissipative systems and has been established as the most general and exact principle of macroscopic continuum physics. For the description of viscous flow systems, Vincze (1971) used the GPDP to derive the equations of thermodynamics. Many other variational principles have already been shown as partial forms of Gyarmati's principle.

The balance equations of the system play a central role in the formulation of Gyarmati's variational principle and, hence, governing Eqs. (2.1) are written in the balance form as

$$
\begin{aligned}
& \nabla \cdot \mathbf{V}=0 \quad \mathbf{V}=\mathbf{i} u+\mathbf{j} v \\
& \rho(\mathbf{V} \cdot \nabla) \mathbf{V}+\nabla \cdot \overline{\bar{P}}=\left(\kappa B_{0}^{2}\right)\left[U_{\infty}-(\mathbf{i} \cdot \mathbf{V})\right] \\
& \rho C_{p}(\mathbf{V} \cdot \nabla) T+\nabla \cdot \mathbf{J}_{q}=\mu\left(u_{y}^{2}\right)-\left(\kappa B_{0}^{2}\right)(\mathbf{i} \cdot \mathbf{V})\left(U_{\infty}-\mathbf{i} \cdot \mathbf{V}\right)
\end{aligned}
$$

These equations represent the mass, momentum and energy balances respectively. Here $\mathbf{i}$ and $\mathbf{j}$ are the unit vectors in the directions of $x$ and $y$ axes, respectively. In Eq. (3.11) $)_{2} \overline{\bar{P}}$ denotes the pressure tensor which can be decomposed as (Gyarmati, 1969)

$$
\overline{\bar{P}}=p \overline{\bar{\delta}}+\overline{\bar{P}}^{v s}
$$

where $p$ is the hydrostatic pressure, $\overline{\bar{\delta}}$ is the unit tensor and $\stackrel{\circ}{\bar{P}}^{v s}$ is the symmetrical part of the viscous pressure tensor whose trace is zero.

In the study of heat transfer and fluid flow problems, the energy picture of Gyarmati's principle is always advantageous over entropy picture. Therefore, the energy dissipation $T \sigma$ is used instead of the entropy production $\sigma$. The energy dissipation for the present system is given by (Gyarmati, 1969)

$$
T \sigma=-J_{q} \frac{\partial \ln T}{\partial y}-P_{12} \frac{\partial u}{\partial y}
$$

where $J_{q}$ is the heat flux and $P_{12}$ is the only component of the momentum flux $\stackrel{\circ}{\bar{P}} v s$, satisfy the constitutive relations connecting the independent fluxes and forces as

$$
J_{q}=-L_{\lambda} \frac{\partial \ln T}{\partial y} \quad P_{12}=-L_{s} \frac{\partial u}{\partial y}
$$

Here $L_{\lambda}=\lambda T$ and $L_{s}=\mu$, where $\lambda$ and $\mu$ are the thermal conductivity and viscosity, respectively. With the help of Eq. (3.14) the dissipation potentials in energy picture are found as follows

$$
T \psi=\frac{1}{2}\left[L_{\lambda}\left(\frac{\partial \ln T}{\partial y}\right)^{2}+L_{s}\left(\frac{\partial u}{\partial y}\right)^{2}\right] \quad T \Phi=\frac{1}{2}\left(R_{\lambda} J_{q}^{2}+R_{s} P_{12}^{2}\right)
$$

where $L_{\lambda}=R_{\lambda}^{-1}$ and $L_{s}=R_{s}^{-1}$. 
Using Eqs. (3.13)-(3.15), Gyarmati's variational principle (3.1) is formulated in the following form

$$
\delta \int_{0}^{l} \int_{0}^{\infty}\left[-J_{q} \frac{\partial \ln T}{\partial y}-P_{12} \frac{\partial u}{\partial y}-\frac{L_{\lambda}}{2}\left(\frac{\partial \ln T}{\partial y}\right)^{2}-\frac{L_{s}}{2}\left(\frac{\partial u}{\partial y}\right)^{2}-\frac{R_{\lambda}}{2} J_{q}^{2}-\frac{R_{s}}{2} P_{12}^{2}\right] d y d x=0
$$

in which $l$ is the representative length of the surface.

\section{Solution procedure}

The system of two dimensional magneto hydrodynamics laminar boundary layer flow over a semi infinite flat plate with viscous dissipation effects in the presence of suction/injection is considered. The velocity and temperature fields inside the respective boundary layers are suitably described by the following functions

$$
\begin{aligned}
& \frac{u}{U_{\infty}}=\frac{2 y}{d_{1}}-\frac{2 y^{3}}{d_{1}^{3}}+\frac{y^{4}}{d_{1}^{4}} \quad \text { for } \quad y<d_{1} \\
& u=U_{\infty} \quad \text { for } y \geqslant d_{1} \\
& \frac{T-T_{\infty}}{T_{0}-T_{\infty}}=1-\frac{2 y}{d_{2}}+\frac{2 y^{3}}{d_{2}^{3}}-\frac{y^{4}}{d_{2}^{4}} \quad \text { for } \quad y<d_{2} \\
& T=T_{\infty} \quad \text { for } y \geqslant d_{2}
\end{aligned}
$$

which satisfy the following compatibility conditions

$$
\begin{aligned}
& y=0 \quad \Rightarrow \quad u=0, \quad v=v_{0}, \quad T=T_{0}, \quad \frac{\partial T}{\partial y}=0 \quad \text { (smooth fit), } \quad \frac{\partial^{2} T}{\partial y^{2}}=0 \\
& y=d_{1} \Rightarrow u=U_{\infty}=\text { const }, \quad \frac{\partial u}{\partial y}=0 \quad \text { (smooth fit), } \quad \frac{\partial^{2} u}{\partial y^{2}}=0 \\
& y=d_{2} \Rightarrow T=T_{\infty}, \quad \frac{\partial T}{\partial y}=0 \quad \text { (smooth fit) }
\end{aligned}
$$

The unknown quantities $d_{1}, d_{2}$ are the extent of the hypothetical hydro dynamical and thermal boundary layer thicknesses respectively. These unknown quantities are to be determined from the present thermodynamic analysis. The smooth fit conditions $\partial u / \partial y=0$ and $\partial T / \partial y=0$ correspond to $P_{12}=0$ and $J_{q}=0$ at their respective edges of the boundary layer. Using boundary conditions (4.2), the transverse velocity component $v$ is obtained from mass balance equation $(3.11)_{1}$ as

$$
v=U_{\infty}\left(\frac{4 y^{5}}{5 d_{1}^{5}}-\frac{3 y^{4}}{2 d_{1}^{4}}+\frac{y^{2}}{d_{1}^{2}}\right) d_{1}^{\prime}+v_{0}
$$

Velocity and temperature functions (4.1) and boundary conditions (4.2) are used in governing Eqs. (3.11), and on direct integration with respect to $y$ with the help of their corresponding smooth fit conditions $u_{y}=0$ and $T_{y}=0$, the momentum flux $P_{12}$ and energy flux $J_{q}$ are obtained. The expression for $P_{12}$ remains the same for any Prandtl number Pr. But the energy flux $J_{q}$ assumes different expressions for $\operatorname{Pr} \leqslant 1$ and $\operatorname{Pr} \geqslant 1$, respectively. When $\operatorname{Pr} \leqslant 1$, the expression for $J_{q}$ in the range $d_{1} \leqslant y \leqslant d_{2}$ is obtained first and the expression for $J_{q}$ in the range $0 \leqslant y \leqslant d_{1}$ is determined subsequently by matching the expressions of the two regions at the interface. The expressions for momentum and the energy fluxes $P_{12}$ and $J_{q}$ are obtained respectively as follows: 
- for $0 \leqslant y \leqslant d_{1}$

$$
\begin{aligned}
& -\frac{P_{12}}{L_{s}}=\frac{U_{\infty}^{2} d_{1}^{\prime}}{\nu}\left(-\frac{4 y^{9}}{45 d_{1}^{9}}+\frac{2 y^{8}}{5 d_{1}^{8}}-\frac{3 y^{7}}{7 d_{1}^{7}}-\frac{11 y^{6}}{15 d_{1}^{6}}+\frac{7 y^{5}}{5 d_{1}^{5}}-\frac{2 y^{3}}{3 d_{1}^{3}}+\frac{101}{1800}\right) \\
& +\frac{v_{0} U_{\infty}}{\nu}\left(\frac{y^{4}}{d_{1}^{4}}-\frac{2 y^{3}}{d_{1}^{3}}+\frac{2 y}{d_{1}}-\frac{7}{10}\right)+\frac{\kappa B_{0}^{2} U_{\infty}}{\rho \nu}\left(\frac{y^{5}}{5 d_{1}^{4}}-\frac{y^{4}}{2 d_{1}^{3}}+\frac{y^{2}}{d_{1}}-y+\frac{7 d_{1}}{30}\right)+\frac{U_{\infty}}{d_{1}}
\end{aligned}
$$

- for $0 \leqslant y \leqslant d_{1}, \operatorname{Pr} \leqslant 1$

$$
\begin{gathered}
-\frac{J_{q}}{L_{\lambda}}=\frac{\operatorname{Pr} U_{\infty}\left(T_{0}-T_{\infty}\right)}{\nu}\left[\left(\frac{4 y^{9}}{9 d_{1}^{4} d_{2}^{5}}-\frac{y^{8}}{d_{1}^{3} d_{2}^{5}}-\frac{3 y^{8}}{4 d_{1}^{4} d_{2}^{4}}+\frac{12 y^{7}}{7 d_{1}^{3} d_{2}^{4}}+\frac{4 y^{6}}{3 d_{1} d_{2}^{5}}+\frac{y^{6}}{3 d_{1}^{4} d_{2}^{2}}\right.\right. \\
\left.-\frac{12 y^{5}}{5 d_{1} d_{2}^{4}}-\frac{4 y^{5}}{5 d_{1}^{3} d_{2}^{2}}+\frac{4 y^{3}}{3 d_{1} d_{2}^{2}}+\frac{d_{1}^{5}}{45 d_{2}^{5}}-\frac{9 d_{1}^{4}}{140 d_{2}^{4}}+\frac{2 d_{1}^{2}}{15 d_{2}^{2}}-\frac{3}{10}\right) d_{2}^{\prime}+\left(-\frac{16 y^{9}}{45 d_{1}^{5} d_{2}^{4}}\right. \\
+\frac{3 y^{8}}{5 d_{1}^{5} d_{2}^{3}}+\frac{3 y^{8}}{4 d_{1}^{4} d_{2}^{4}}-\frac{9 y^{7}}{7 d_{1}^{4} d_{2}^{3}}-\frac{2 y^{6}}{3 d_{1}^{2} d_{2}^{4}}-\frac{4 y^{6}}{15 d_{1}^{5} d_{2}}+\frac{3 y^{5}}{5 d_{1}^{4} d_{2}}+\frac{6 y^{5}}{5 d_{1}^{2} d_{2}^{3}}-\frac{2 y^{3}}{3 d_{1}^{2} d_{2}} \\
\left.\left.+\frac{49 d_{1}^{4}}{180 d_{2}^{4}}-\frac{18 d_{1}^{3}}{35 d_{2}^{3}}+\frac{d_{1}}{3 d_{2}}\right) d_{1}^{\prime}+\frac{v_{0}}{U_{\infty}}\left(-\frac{y^{4}}{d_{2}^{4}}+\frac{2 y^{3}}{d_{2}^{3}}-\frac{2 y}{d_{2}}+\frac{d_{1}^{4}}{d_{2}^{4}}-\frac{2 d_{1}^{3}}{d_{2}^{3}}+\frac{2 d_{1}}{d_{2}}\right)\right] \\
+\frac{\operatorname{Pr} U_{\infty}^{2}}{C_{p}}\left(-\frac{16 y^{7}}{7 d_{1}^{8}}+\frac{8 y^{6}}{d_{1}^{7}}-\frac{36 y^{5}}{5 d_{1}^{6}}-\frac{4 y^{4}}{d_{1}^{5}}+\frac{8 y^{3}}{d_{1}^{4}}-\frac{4 y}{d_{1}^{2}}+\frac{52}{35 d_{1}}\right) \\
+\frac{\operatorname{Pr} \kappa B_{0}^{2} U_{\infty}^{2}}{\nu \rho C_{p}}\left(-\frac{y^{9}}{9 d_{1}^{8}}+\frac{y^{8}}{2 d_{1}^{7}}-\frac{4 y^{7}}{7 d_{1}^{6}}-\frac{2 y^{6}}{3 d_{1}^{5}}+\frac{9 y^{5}}{5 d_{1}^{4}}-\frac{y^{4}}{2 d_{1}^{3}}-\frac{4 y^{3}}{3 d_{1}^{2}}+\frac{y^{2}}{d_{1}}-\frac{37 d_{1}}{315}\right)
\end{gathered}
$$

- for $d_{1} \leqslant y \leqslant d_{2}, \operatorname{Pr} \leqslant 1$

$$
-\frac{J_{q}}{L_{\lambda}}=\frac{\operatorname{Pr} U_{\infty}\left(T_{0}-T_{\infty}\right) d_{2}^{\prime}}{\nu}\left(\frac{4 y^{5}}{5 d_{2}^{5}}-\frac{3 y^{4}}{2 d_{2}^{4}}+\frac{y^{2}}{d_{2}^{2}}-\frac{3}{10}\right)
$$

- for $0 \leqslant y \leqslant d_{2}, \operatorname{Pr} \geqslant 1$

$$
\begin{aligned}
& -\frac{J_{q}}{L_{\lambda}}=\frac{\operatorname{Pr} U_{\infty}\left(T_{0}-T_{\infty}\right)}{\nu}\left[\left(\frac{4 y^{9}}{9 d_{1}^{4} d_{2}^{5}}-\frac{y^{8}}{d_{1}^{3} d_{2}^{5}}-\frac{3 y^{8}}{4 d_{1}^{4} d_{2}^{4}}+\frac{12 y^{7}}{7 d_{1}^{3} d_{2}^{4}}+\frac{4 y^{6}}{3 d_{1} d_{2}^{5}}+\frac{y^{6}}{3 d_{1}^{4} d_{2}^{2}}\right.\right. \\
& \left.-\frac{4 y^{5}}{5 d_{1}^{3} d_{2}^{2}}-\frac{12 y^{5}}{5 d_{1} d_{2}^{4}}+\frac{4 y^{3}}{3 d_{1} d_{2}^{2}}-\frac{d_{2}^{4}}{36 d_{1}^{4}}+\frac{3 d_{2}^{3}}{35 d_{1}^{3}}-\frac{4 d_{2}}{15 d_{1}}\right) d_{2}^{\prime}+\left(-\frac{16 y^{9}}{45 d_{1}^{5} d_{2}^{4}}+\frac{3 y^{8}}{5 d_{1}^{5} d_{2}^{3}}\right. \\
& +\frac{3 y^{8}}{4 d_{1}^{4} d_{2}^{4}}-\frac{9 y^{7}}{7 d_{1}^{4} d_{2}^{3}}-\frac{2 y^{6}}{3 d_{1}^{2} d_{2}^{4}}-\frac{4 y^{6}}{15 d_{1}^{5} d_{2}}+\frac{6 y^{5}}{5 d_{1}^{2} d_{2}^{3}}+\frac{3 y^{5}}{5 d_{1}^{4} d_{2}}-\frac{2 y^{3}}{3 d_{1}^{2} d_{2}}+\frac{d_{2}^{5}}{45 d_{1}^{5}} \\
& \left.\left.-\frac{9 d_{2}^{4}}{140 d_{1}^{4}}+\frac{2 d_{2}^{2}}{15 d_{1}^{2}}\right) d_{1}^{\prime}+\frac{v_{0}}{U_{\infty}}\left(-\frac{y^{4}}{d_{2}^{4}}+\frac{2 y^{3}}{d_{2}^{3}}-\frac{2 y}{d_{2}}+1\right)\right]-\frac{\operatorname{Pr} U_{\infty}^{2}}{C_{p}}\left(\frac{16 y^{7}}{7 d_{1}^{8}}-\frac{8 y^{6}}{d_{1}^{7}}\right. \\
& \left.+\frac{36 y^{5}}{5 d_{1}^{6}}+\frac{4 y^{4}}{d_{1}^{5}}-\frac{8 y^{3}}{d_{1}^{4}}+\frac{4 y}{d_{1}^{2}}-\frac{16 d_{2}^{7}}{7 d_{1}^{8}}+\frac{8 d_{2}^{6}}{d_{1}^{7}}-\frac{36 d_{2}^{5}}{5 d_{1}^{6}}-\frac{4 d_{2}^{4}}{d_{1}^{5}}+\frac{8 d_{2}^{3}}{d_{1}^{4}}-\frac{4 d_{2}}{d_{1}^{2}}\right) \\
& +\frac{\operatorname{Pr} \kappa B_{0}^{2} U_{\infty}^{2}}{\nu \rho C_{p}}\left(-\frac{y^{9}}{9 d_{1}^{8}}+\frac{y^{8}}{2 d_{1}^{7}}-\frac{4 y^{7}}{7 d_{1}^{6}}-\frac{2 y^{6}}{3 d_{1}^{5}}+\frac{9 y^{5}}{5 d_{1}^{4}}-\frac{y^{4}}{2 d_{1}^{3}}-\frac{4 y^{3}}{3 d_{1}^{2}}+\frac{y^{2}}{d_{1}}+\frac{d_{2}^{9}}{9 d_{1}^{8}}\right. \\
& \left.-\frac{d_{2}^{8}}{2 d_{1}^{7}}+\frac{4 d_{2}^{7}}{7 d_{1}^{6}}+\frac{2 d_{2}^{6}}{3 d_{1}^{5}}-\frac{9 d_{2}^{5}}{5 d_{1}^{4}}+\frac{d_{2}^{4}}{2 d_{1}^{3}}+\frac{4 d_{2}^{3}}{3 d_{1}^{2}}-\frac{d_{2}^{2}}{d_{1}}\right)
\end{aligned}
$$

The prime indicates differentiation with respect to $x$. Using the expressions of $P_{12}$ and $J_{q}$ together with velocity and temperature functions (4.1), variational principle (3.16) is formulated independently for $\operatorname{Pr} \leqslant 1$ and $\operatorname{Pr} \geqslant 1$, respectively. After performing the integration with respect to $y$, one can obtain the variational principle in the following forms 


$$
\begin{array}{ll}
\delta \int_{0}^{l} L_{1}\left[d_{1}, d_{2}, d_{1}^{\prime}, d_{2}^{\prime}\right] d x=0 & \operatorname{Pr} \leqslant 1 \\
\delta \int_{0}^{l} L_{2}\left[d_{1}, d_{2}, d_{1}^{\prime}, d_{2}^{\prime}\right] d x=0 & \operatorname{Pr} \geqslant 1
\end{array}
$$

where $L_{1}, L_{2}$ are the Lagrangian densities of the principle. The variation is carried out with respect to the independent parameters $d_{1}$ and $d_{2}$. These variational principles, (4.8), are found identical when $d_{1}=d_{2}$. The Euler-Lagrange equations corresponding to these variational parameters are

$$
\frac{\partial L_{1,2}}{\partial d_{1}}-\frac{d}{d x}\left(\frac{\partial L_{1,2}}{\partial d_{1}^{\prime}}\right)=0 \quad \frac{\partial L_{1,2}}{\partial d_{2}}-\frac{d}{d x}\left(\frac{\partial L_{1,2}}{\partial d_{2}^{\prime}}\right)=0 \quad(\operatorname{Pr} \leqslant 1, \operatorname{Pr} \geqslant 1)
$$

where $L_{1,2}$ represents the Lagrangian densities $L_{1}$ and $L_{2}$ respectively. Equations (4.9) are second order ordinary differential equations in terms of $d_{1}$ and $d_{2}$. The procedure for solving (4.9) can be considerably simplified by introducing the non dimensional boundary layer thicknesses $d_{1}^{*}$ and $d_{2}^{*}$ and are given by

$$
d_{1}=d_{1}^{*} \sqrt{\frac{\nu x}{U_{\infty}}} \quad d_{2}=d_{2}^{*} \sqrt{\frac{\nu x}{U_{\infty}}}
$$

Variational principles (4.8) are subject to transformations (4.10). The resulting EulerLagrange equations are obtained as simple polynomial equations

$$
\frac{\partial L_{1,2}}{\partial d_{1}^{*}}=0 \quad \frac{\partial L_{1,2}}{\partial d_{2}^{*}}=0 \quad(\operatorname{Pr} \leqslant 1, \operatorname{Pr} \geqslant 1)
$$

The coefficients of Eqs. (4.11) depend on the independent parameters $\operatorname{Pr}, H, \xi$ and Ec, where $\operatorname{Pr}=\nu / \alpha$ (Prandtl number), $H=v_{0} \sqrt{x /\left(\nu U_{\infty}\right)}$ (suction/injection parameter), $\xi=\kappa B_{0}^{2} x /\left(\rho U_{\infty}\right)$ (magnetic parameter) and $\mathrm{Ec}=U_{\infty}^{2} /\left[C_{p}\left(T_{0}-T_{\infty}\right)\right]$ (Eckert number).

In the present analysis, suction and injection are represented by $H<0$ and $H>0$ respectively. Equation $(4.11)_{1}$ is a simple polynomial equation in terms of boundary layer thickness whose coefficients depend on the parameters $H$ and $\xi$. This equation is solved for any combination of $H$ and $\xi$, and the corresponding hydro dynamical boundary layer thickness $d_{1}^{*}$ is obtained as the only positive root.

Equation $(4.11)_{2}$ is solved for any values of $\operatorname{Pr}, H, \xi$, and Ec and it is found that for any value of $\operatorname{Pr}$ there corresponds only one real root $d_{2}^{*}$. After obtaining the values of $d_{1}^{*}$ and $d_{2}^{*}$ for given values of $\operatorname{Pr}, H, \xi$ and $\mathrm{Ec}$, the values of velocity, temperature profiles, skin friction (shear stress) and heat transfer (Nusselt number) are calculated with the help of the following expressions

$$
\eta=y \sqrt{\frac{U_{\infty}}{\nu x}} \quad \tau_{w}=\sqrt{\frac{\nu x}{U_{\infty}^{3}}}\left(-\frac{P_{12}}{L_{s}}\right)_{y=0}
$$

and

$$
\mathrm{Nu}_{l}=\sqrt{\frac{\nu x}{U_{\infty}}\left(T_{0}-T_{\infty}\right)^{2}}\left(\frac{J_{q}}{L_{\lambda}}\right)_{y=0}
$$




\section{Analysis of the results}

The main and important characteristics of the problem analyzed are skin friction and heat transfer values. The thermal energy equation has been solved for two cases $d_{1}^{*} \leqslant d_{2}^{*}(\operatorname{Pr} \leqslant 1)$ and $d_{1}^{*} \geqslant d_{2}^{*}(\operatorname{Pr} \geqslant 1)$. These two independent analyses yield solutions matching at $\operatorname{Pr}=1$. It is found that both the analyses lead to satisfactory results in the respective ranges of $\mathrm{Pr}$.

Table 1 exhibits the skin friction values for various values of suction/injection parameter $H$ ranging from -0.50 to 0.50 when $\xi=0$. It is observed that the hydrodynamical boundary layer thickness $d_{1}^{*}$ increases with $H$ while the skin friction values decrease with $H$.

Table 1. Skin friction for various values of $H$ when $\xi=0$

\begin{tabular}{|c|c|c|}
\hline$H$ & $d_{1}^{*}$ & $\tau_{w}$ \\
\hline \hline-0.50 & 3.2028266677 & 0.7520812815 \\
\hline-0.45 & 3.3763533516 & 0.7059029983 \\
\hline-0.40 & 3.5631288866 & 0.6606177694 \\
\hline-0.35 & 3.7638383130 & 0.6162827997 \\
\hline-0.30 & 3.9790701394 & 0.5729500181 \\
\hline-0.25 & 4.2092882548 & 0.5306637731 \\
\hline-0.20 & 4.4548080980 & 0.4894586742 \\
\hline-0.15 & 4.7157800455 & 0.4493578218 \\
\hline-0.10 & 4.9921823020 & 0.4103716428 \\
\hline-0.05 & 5.2838243570 & 0.3724974836 \\
\hline 0.00 & 5.5903605565 & 0.3357200069 \\
\hline 0.05 & 5.9113119209 & 0.3000123222 \\
\hline 0.10 & 6.2460933581 & 0.2653376883 \\
\hline 0.15 & 6.5940430695 & 0.2316515653 \\
\hline 0.20 & 6.9544512195 & 0.1989037868 \\
\hline 0.25 & 7.3265856367 & 0.1670406519 \\
\hline 0.30 & 7.7097131998 & 0.1360067937 \\
\hline 0.35 & 8.1031163960 & 0.1057467373 \\
\hline 0.40 & 8.5061051918 & 0.0762061206 \\
\hline 0.45 & 8.9180247739 & 0.0473325882 \\
\hline 0.50 & 9.3382599143 & 0.0190763965 \\
\hline & &
\end{tabular}

Soundalgekar and Takhar (1977) gave the non-dimensional skin friction values for $\xi=5$ and $\xi=6$ as 2.01 and 2.255, respectively. Similarly, Ingham (1979) computed these values as 2.235 and 2.449 for the non-dimensional skin friction for $\xi=5$ and $\xi=6$ respectively, where as the present computed values are 2.2815 and 2.4951 for the cases $\xi=5$ and $\xi=6$, respectively.

Tables 2 and 3 display the heat transfer values for various values of $\mathrm{Ec}, \xi$ for the given values of $\operatorname{Pr}=0.733$ and $\operatorname{Pr}=1$, when $H=0$ respectively. From these two tables, the obtained heat transfer values from the present thermodynamic analysis are compared with Watanabe and Pop (1994) for the cases $\mathrm{Ec}=0, \mathrm{Ec}=0.5$ and $\mathrm{Ec}=1.0$. The comparison of the present heat transfer values with those of Watanabe and Pop (1994) is excellent with a very high order of accuracy. From these two tables, it is noted that the heat transfer values are increasing with the magnetic parameter $\xi$ and decreasing with Ec.

In Table 4, the heat transfer values are compared with various values of $\operatorname{Pr}(\operatorname{Pr} \leqslant 1$ and $\operatorname{Pr} \geqslant 1)$ when $\mathrm{Ec}=H=\xi=0$. It is also observed that the heat transfer increases with the values of Prandtl number. Since the higher Prandtl number has very low thermal conductivity, the increase in the heat transfer rate is rapid. This means that the variation of the local Nusselt 
Table 2. Local Nusselt number for various values of Ec, when $\operatorname{Pr}=0.733$ and $H=0$

\begin{tabular}{|c|c|c|c|c|c|c|}
\hline \multicolumn{4}{|c|}{ Present results } & \multicolumn{3}{c|}{ Watanabe and Pop (1994) } \\
\hline$\xi$ & Ec $=0.0$ & Ec $=0.5$ & Ec $=1.0$ & Ec $=0.0$ & Ec $=0.5$ & Ec $=1.0$ \\
\hline \hline 0.0 & 0.2997214322 & 0.2104916973 & 0.1594322283 & 0.29755 & 0.21075 & 0.12395 \\
\hline 0.5 & 0.3509466982 & 0.2715673472 & 0.1917651052 & 0.35699 & 0.28285 & 0.20871 \\
\hline 1.0 & 0.3786430015 & 0.3051396337 & 0.2113702944 & 0.38336 & 0.30532 & 0.22857 \\
\hline 1.5 & 0.3961867294 & 0.3197513425 & 0.2381053043 & 0.39959 & 0.31986 & 0.24122 \\
\hline 2.0 & 0.4084325641 & 0.3200609301 & 0.2499078214 & 0.41091 & 0.33011 & 0.25022 \\
\hline
\end{tabular}

Table 3. Local Nusselt number for various values of Ec, when $\operatorname{Pr}=1$ and $H=0$

\begin{tabular}{|c|c|c|c|c|c|c|}
\hline \multicolumn{4}{|c|}{ Present results } & \multicolumn{3}{c|}{ Watanabe and Pop (1994) } \\
\hline$\xi$ & Ec $=0.0$ & Ec $=0.5$ & Ec $=1.0$ & Ec $=0.0$ & Ec $=0.5$ & Ec $=1.0$ \\
\hline \hline 0.0 & 0.3342775444 & 0.2432735955 & 0.1590535671 & 0.33206 & 0.24904 & 0.16603 \\
\hline 0.5 & 0.3911204994 & 0.2845735809 & 0.1972096839 & 0.40280 & 0.30212 & 0.20144 \\
\hline 1.0 & 0.4232045708 & 0.2971271948 & 0.2105439783 & 0.43446 & 0.32519 & 0.21727 \\
\hline 1.5 & 0.4444082310 & 0.3006179108 & 0.2264237472 & 0.45413 & 0.34005 & 0.22710 \\
\hline 2.0 & 0.4596797063 & 0.3498462661 & 0.2296600733 & 0.46798 & 0.35052 & 0.23401 \\
\hline
\end{tabular}

Table 4. Local Nusselt number for various values of $\operatorname{Pr}$ when $\mathrm{Ec}=H=\xi=0$

\begin{tabular}{|c|c|c|c|}
\hline $\operatorname{Pr}$ & $\begin{array}{c}\text { Present results } \\
\mathrm{Nu}_{l}\end{array}$ & $\begin{array}{c}\text { Lin and Lin (1987) } \\
\mathrm{Nu}_{l}\end{array}$ & $\begin{array}{c}\text { Yih (1999) } \\
\mathrm{Nu}_{l}\end{array}$ \\
\hline \hline 0.0001 & 0.005637108 & 0.005588 & 0.005590 \\
\hline 0.001 & 0.017773426 & 0.017316 & 0.017316 \\
\hline 0.01 & 0.054742313 & 0.051590 & 0.051589 \\
\hline 0.1 & 0.147754551 & 0.140032 & 0.140034 \\
\hline 1 & 0.334277544 & 0.332058 & 0.332057 \\
\hline 10 & 0.738452128 & 0.728148 & 0.728141 \\
\hline 100 & 1.599967934 & 1.57186 & 1.571831 \\
\hline 1000 & 3.451398141 & 3.38710 & 3.387083 \\
\hline 10000 & 7.437892045 & 7.29742 & 7.297402 \\
\hline
\end{tabular}

number is more sensitive to the lager Prandtl number than the smaller one. From this table, it is evidently clear that the present results are in good agreement with Lin and Lin (1987) and Yih (1999).

For heat transfer, Soundalgekar and Takhar (1977) presented the values as 0.4180 and 0.4241 for $\xi=5$ and $\xi=6$ when $\mathrm{Ec}=0.01$ and $\operatorname{Pr}=0.733$, respectively. Similarly, Ingham (1979) gave 0.4381 and 0.4430 for $\xi=5$ and $\xi=6$ when $\mathrm{Ec}=0.01$ and $\operatorname{Pr}=0.733$ respectively, where as the present result gives 0.4385 and 0.4433 , respectively.

Tables 5 and 6 present the temperature distributions, $\theta=\left(T-T_{\infty}\right) /\left(T_{0}-T_{\infty}\right)$ for $\xi=5$ and 6 when $\mathrm{Ec}=0.01, H=0$ and $\operatorname{Pr}=0.733$, respectively. Thus, the variational solution obtained with the help of Governing Principle of Dissipative Processes is quite in agreement with the exact numerical results of Soundalgekar and Takhar (1977) and Ingham (1979) even though Soundalgekar and Takhar (1977) omitted the terms from the full boundary layer equations.

Figure 1 represents the skin friction values as a function of $\xi$ for different values of $H$. From this figure, it is observed that the values of skin friction increases with the magnetic parameter $\xi$ irrespective of the suction and injection parameter $H$. One can also note that, when the suction and injection speed $H$ increases, the values of skin friction decreases. 
Table 5. Temperature distribution for $\xi=5$ when $\mathrm{Ec}=0.01, H=0, \operatorname{Pr}=0.733$

\begin{tabular}{|c|c|c|c|}
\hline$\eta$ & Present results & Ingham (1979) & Soundalgekar and Takhar (1977) \\
& $\theta$ & $\theta$ & $\theta$ \\
\hline \hline 0.00 & 1.000000000 & 1.0000 & 1.0000 \\
\hline 0.28 & 0.876740762 & 0.8771 & 0.8823 \\
\hline 0.56 & 0.753881748 & 0.7554 & 0.7655 \\
\hline 0.84 & 0.633920705 & 0.6382 & 0.6520 \\
\hline 1.12 & 0.519536384 & 0.5284 & 0.5442 \\
\hline 1.40 & 0.413224176 & 0.4285 & 0.4444 \\
\hline 1.68 & 0.316933549 & 0.3400 & 0.3543 \\
\hline 1.96 & 0.231127979 & 0.2640 & 0.2754 \\
\hline 2.24 & 0.181110807 & 0.2003 & 0.2082 \\
\hline 2.52 & 0.104934854 & 0.1486 & 0.1527 \\
\hline
\end{tabular}

Table 6. Temperature distribution for $\xi=6$ when $\mathrm{Ec}=0.01, H=0, \operatorname{Pr}=0.733$

\begin{tabular}{|c|c|c|c|}
\hline$\eta$ & $\begin{array}{c}\text { Present results } \\
\theta\end{array}$ & $\begin{array}{c}\text { Ingham (1979) } \\
\theta\end{array}$ & $\begin{array}{c}\text { Soundalgekar and Takhar (1977) } \\
\theta\end{array}$ \\
\hline \hline 0.00 & 1.000000000 & 1.0000 & 1.0000 \\
\hline 0.28 & 0.875276392 & 0.8752 & 0.8805 \\
\hline 0.56 & 0.751013068 & 0.7518 & 0.7620 \\
\hline 0.84 & 0.629907409 & 0.6334 & 0.6472 \\
\hline 1.12 & 0.514711507 & 0.5229 & 0.5385 \\
\hline 1.40 & 0.407935204 & 0.4227 & 0.4302 \\
\hline 1.68 & 0.311228464 & 0.3344 & 0.3481 \\
\hline 1.96 & 0.222568501 & 0.2588 & 0.2695 \\
\hline 2.24 & 0.124904812 & 0.1957 & 0.2029 \\
\hline
\end{tabular}

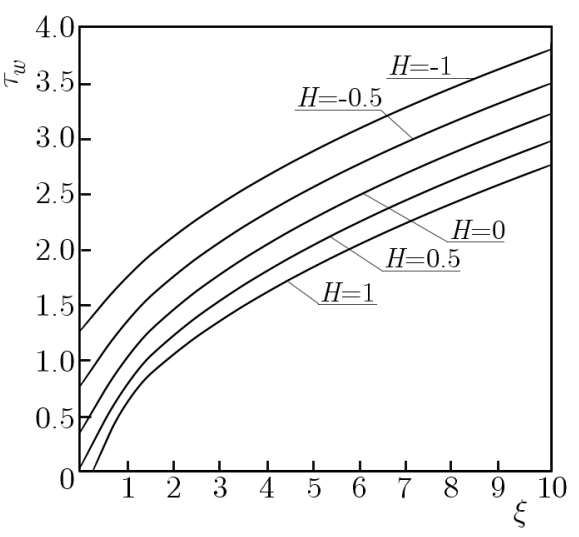

Fig. 1. Skin friction values as a function of $\xi$ for different values of $H$

Figures 2a-2c represent the velocity profile inside the boundary layer for different values of $H$ when $\xi=0.1, \xi=0.2$ and $\xi=0.3$, respectively. From these figures, it can be easily observed that the non-dimensional velocity increases from 0 to 1 and the increase is rapid in the case of suction.

Figure 3 presents the velocity profile for $\xi=5$ and 6 when $H=0$. From this figure it is clearly proved that the present result is well comparable with the results of Ingham (1979) and Soundalgekar and Takhar (1977) and the comparison is excellent. Also it is found that an increase in the magnetic parameter $\xi$ increases the velocity profile. 

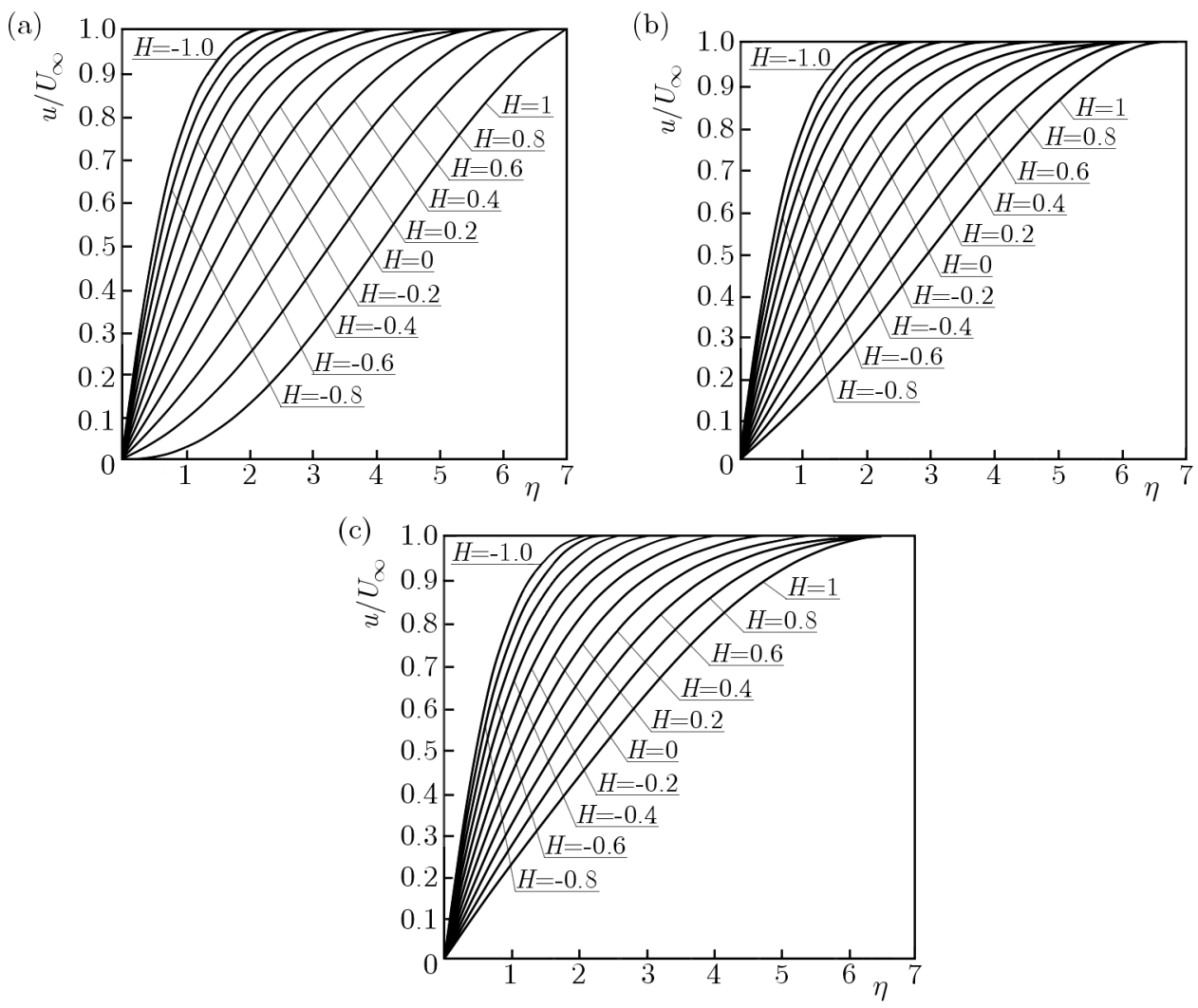

Fig. 2. Velocity profile for different values of $H$ : (a) $\xi=0.1$, (b) $\xi=0.2$, (c) $\xi=0,3$

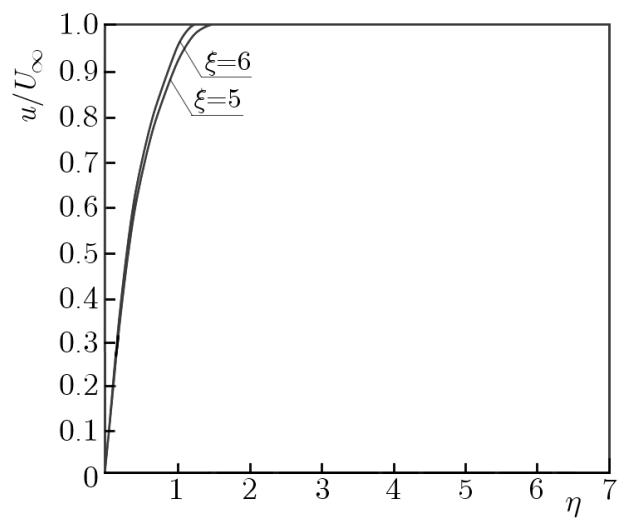

Fig. 3. Velocity profile for $\xi=5$ and $\xi=6$ when $H=0$

The temperature profiles for different values of $\operatorname{Pr}, \mathrm{Ec}$ and $H$ when $\xi=0.2$ are exhibited in Figs. 4 and 5. From these figures it can be interpreted that there is a rapid increase in the temperature in the case of injection and also due to the heat created by the effect of viscous dissipation.

Figures 6 and 7 explain the variation of the local Nusselt number for various values of $\xi$, Pr, Ec and $H$. In the present investigation, the value of the Eckert number is considered as very small for all incompressible fluid flows.

From these figures, it is observed that the rate of heat transfer decreases due to heat generated by the viscous dissipation effect, and it is also observed that the heat transfer increases with $\mathrm{Pr}$ in the case of suction and decreases with $\operatorname{Pr}$ in the case of injection. Contrarily, the heat transfer decreases with $\xi$ in the case of suction and increases with $\xi$ in the case of injection. 

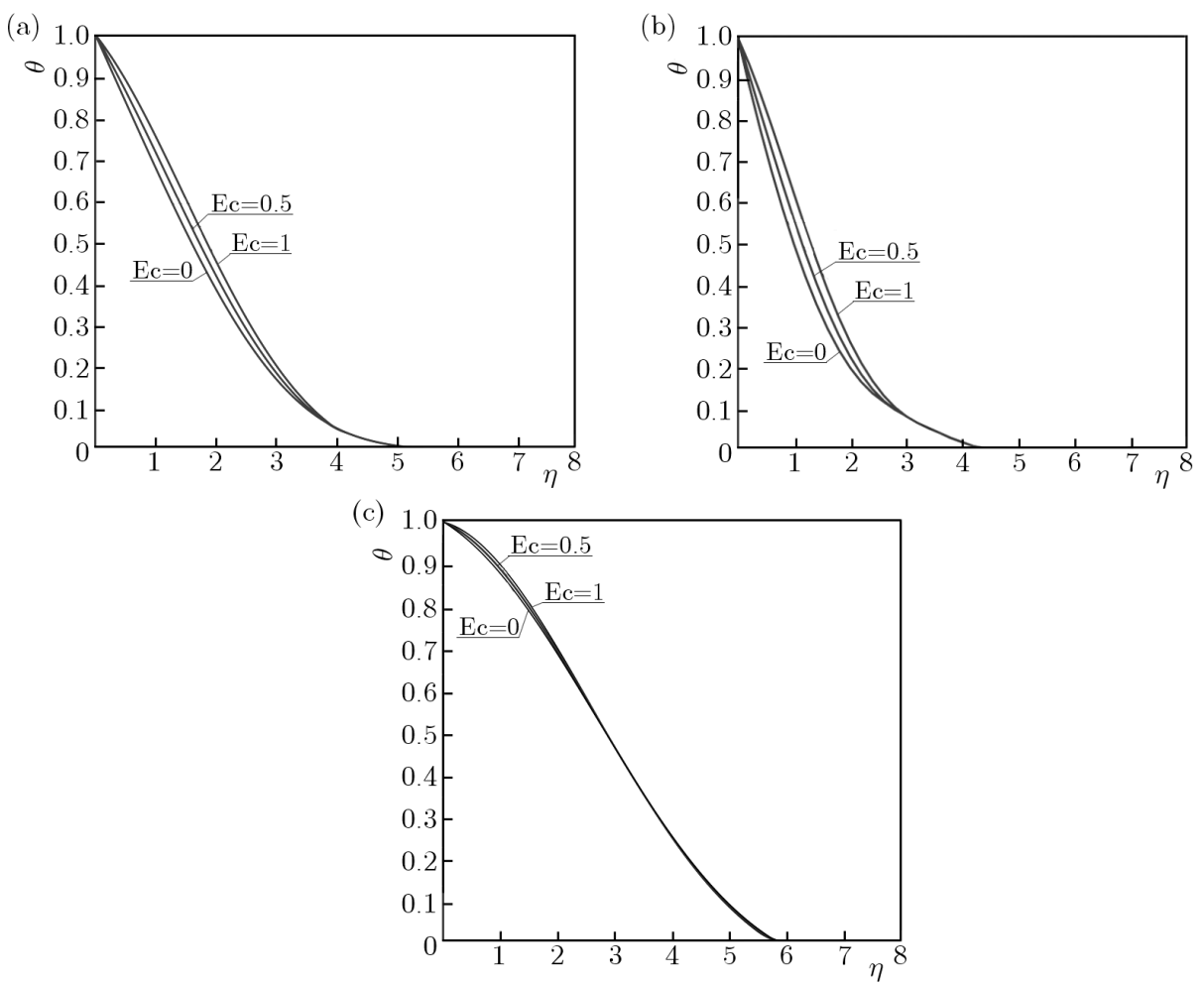

Fig. 4. Temperature profile for $\xi=0.2, \operatorname{Pr}=0.733, \mathrm{Ec}=0,0.5$ and 1.0: (a) $H=0$, (b) $H=-0.5$, (c) $H=0.5$

(a)

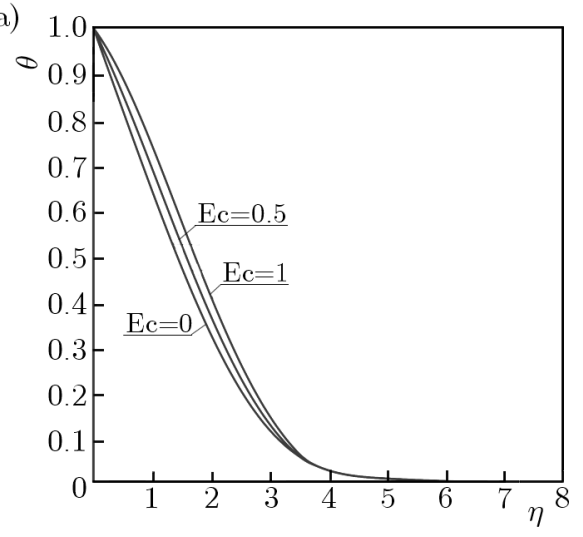

(b)

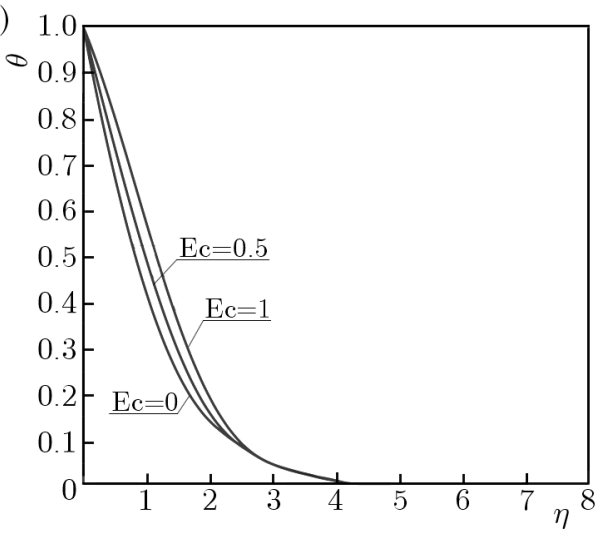

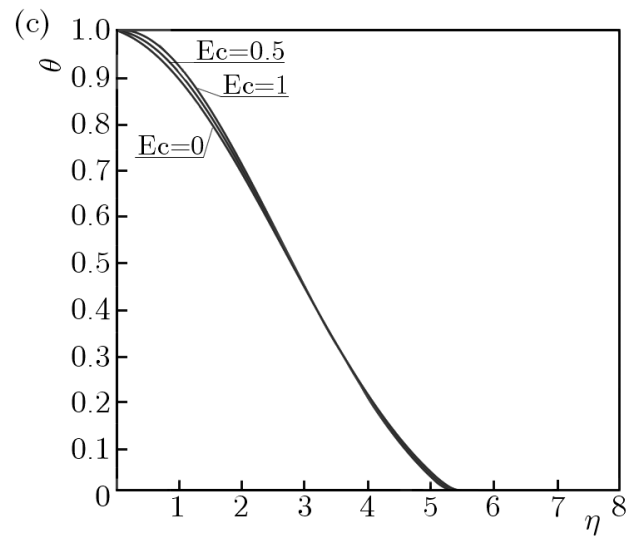

Fig. 5. Temperature profile for $\xi=0.2, \operatorname{Pr}=1, \mathrm{Ec}=0,0.5$ and 1.0: (a) $H=0$, (b) $H=-0.5$, (c) $H=0.5$ 
(a)

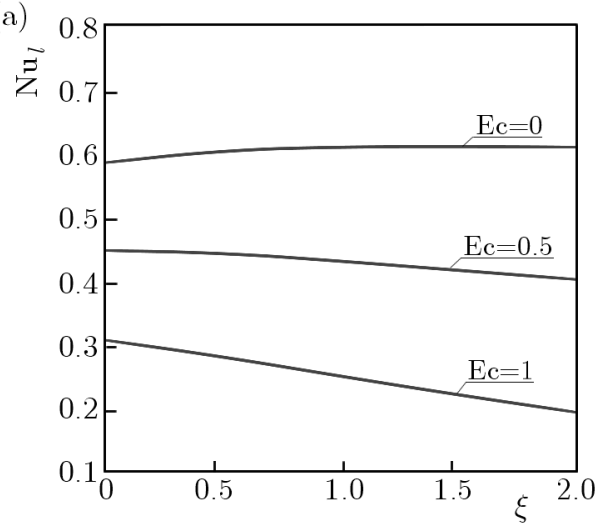

(b)

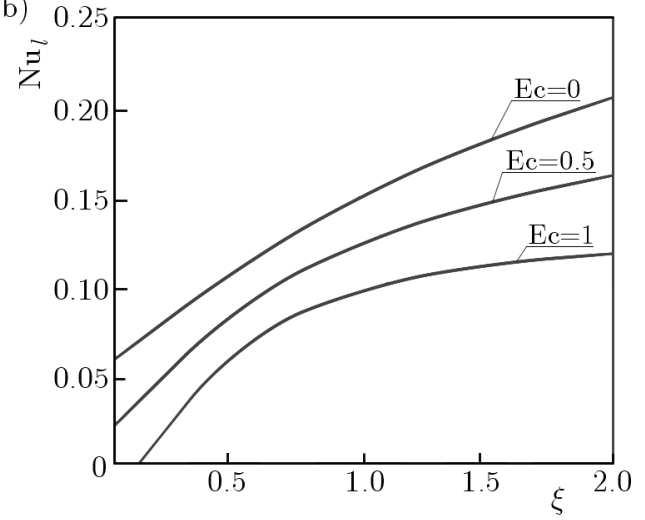

Fig. 6. Variation of the local Nusselt number as a function of $\xi$ for $\operatorname{Pr}=0.733$, Ec $=0,0.5$ and 1.0: (a) $H=-0.5$, (b) $H=0.5$

(a)

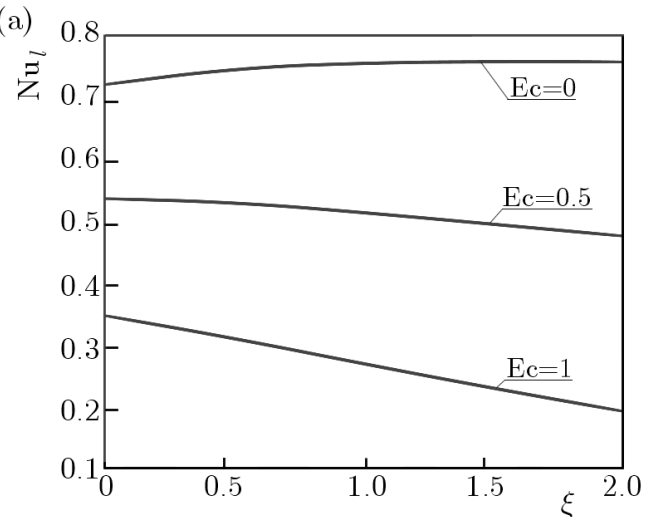

(b)

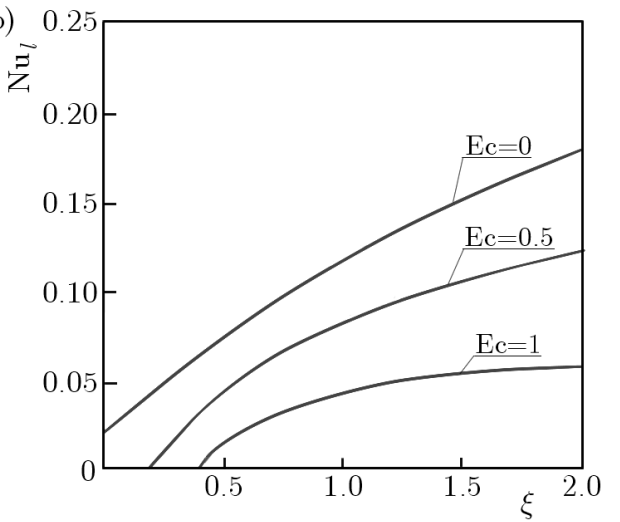

Fig. 7. Variation of the local Nusselt number as a function of $\xi$ for $\operatorname{Pr}=1, \mathrm{Ec}=0,0.5$ and 1.0:

(a) $H=-0.5$, (b) $H=0.5$

\section{Conclusion}

This work deals with the effects of transverse magnetic field, viscous dissipation, skin friction and surface heat transfer over a semi infinite flat plate. The governing partial differential equations, (4.11), are reduced as coupled polynomial equations in non dimensional boundary layer thicknesses $d_{1}^{*}, d_{2}^{*}$, and the coefficients of these equations depent on the independent parameters $\xi, \operatorname{Pr}, \mathrm{Ec}$ and $H$. These equations offer a practicing engineer a rapid way of obtaining shear stress and heat transfer for any combinations of $\xi$, Pr, Ec and $H$. The great advantage involved in the present technique is that the results are obtained with a high order of accuracy and the amount of calculation is certainly less when compared with more conventional methods. Hence the practicing engineers and scientists can employ this unique approximate technique as a powerful tool for solving boundary layer flow and heat transfer problems.

\section{References}

1. Anjali Devi S.P., Kandasamy R., 2003, Thermal stratification effects on nonlinear MHD laminar boundary layer flow over a wedge with suction/injection, International Communications in Heat and Mass Transfer, 30, 717-725

2. Ariel P.D., 1994, Hiemenz flow in hydromagnetics, Acta Mechanica, 103, 31-43 
3. Снамкна A.J., 1998, Hydromagnetic mixed convection stagnation flow with suction and blowing, International Communications in Heat and Mass Transfer, 25, 417-426

4. Chandrasekar M., 1998, Thermodynamical modeling of boundary layer flow with suction and injection, ASME Journal of Applied Mechanics, 65, 3, 764-768

5. Chandrasekar M., 2003, Analytical study of heat transfer on boundary layer flow with suction and injection, Heat and Mass Transfer, 30, 157-165

6. Chandrasekar M., Baskaran S., 2006, Modeling and analytical solution to heat transfer and boundary layer flow with suction and injection, Journal of Non-Equilibrium Thermodynamics, 31, 153-171

7. Chandrasekar M., Baskaran S., 2007, Thermodynamical modeling of viscous dissipation in magnetohydrodynamic flow, Theoretical and Applied Mechanics, 34, 3, 197-219

8. Gyarmati I., 1969, On the governing principle of dissipative processes and its extension to non linear problems, Annals of Physics, 23, 353-378

9. Gyarmati I., 1970, Non Equilibrium Thermodynamics: Field Theory and Variational Principles, Springer-Verlag, Berlin

10. Hossain M.A., 1992, Viscous and joule heating effects on MHD free convection flow with variable plate temperature, International Journal of Heat and Mass Transfer, 35, 3485-3487

11. Ingham D.B., 1979, MHD flows in the presence of a transverse magnetic field, Nuclear Engineering and Design, 52, 325-329

12. LiN H.T., LiN L.K., 1987, Similarity solution for laminar forced convection heat transfer from wedges to fluids of any Prandtl number, International Journal of Heat and Mass Transfer, 30, $1111-1118$

13. Onsagar L., 1931, Reciprocal relations in irreversible processes-I, Physical Review, 37, 405-426

14. Onsagar L., 1931, Reciprocal relations in irreversible processes-II, Physical Review, 38, 2265-2279

15. RAptis A.A., 1991, MHD Asymmetric flow in the presence of diffusion, Wärme-und Stoffübertragung, 26, 193-194

16. Soundalgekar V.M., TAkHar H.S., 1977, On MHD flow and heat transfer over a semi-infinite plate under transverse magnetic field, Nuclear Engineering and Design, 42, 233-236

17. Sparrow E.M., Eckert E.R.G., Minkowycz W.J., 1963, Transpiration cooling in a MHD stagnation point flow, Applied Scientific Research, A11, 125-147

18. Vincze G., 1971, Deduction of the quasi-linear transport equations of hydrodynamics from the Gyarmati's principle, Annals of Physics, 27, 225-236

19. Watanabe T., 1978, Magnetohydrodynamic stability of boundary layers along a flat plate in the presence of a transverse magnetic field, ZAMM, 58, 555-560

20. Watanabe T., 1986, Magnetohydrodynamic stability of boundary layers along a flat plate with pressure gradient, Acta Mechanica, 65, 41-50

21. Watanabe T., 1990, Thermal boundary layers over a wedge with uniform suction/injection in forced flow, Acta Mechanica, 83, 119-126

22. Watanabe T., Pop I., 1993, Magnetohydrodynamic free convection flow over wedge in the presence of a transverse magnetic field, International Communications in Heat and Mass Transfer, 20, 871-881

23. Watanabe T., Pop I., 1994, Thermal boundary layer in Magnetohydrodynamic flow over a flat plate in the presence of a transverse magnetic field, Acta Mechanica, 105, 233-238 
24. Yiн K.A., 1998, Uniform suction/blowing effect on forced convection about a wedge: uniform heat flux, Acta Mechanica, 128, 173-181

25. YIH K.A., 1999, MHD forced convection flow adjacent to a non-isothermal wedge, International Communications in Heat and Mass Transfer, 26, 6, 819-827

Manuscript received April 16, 2013; accepted for print July 22, 2014 